\title{
Direct Evidence for a Surface and Bulk Specific Response in the Sum-Frequency Generation Spectrum of the Water Bend Vibration
}

Carolyn Moll ( $\sim$ cmoll@amol.nl )

AMOLF https://orcid.org/0000-0001-6041-5898

Jan Versluis

AMOLF

Huib Bakker

AMOLF

\section{Article}

Keywords: water bend vibration, spectroscopic studies, aqueous surfaces

Posted Date: February 10th, 2021

DOl: https://doi.org/10.21203/rs.3.rs-198452/v1

License: (c) (i) This work is licensed under a Creative Commons Attribution 4.0 International License.

Read Full License 


\title{
Direct Evidence for a Surface and Bulk Specific Response in the Sum-Frequency Generation Spectrum of the Water Bend Vibration
}

\author{
Carolyn J. Moll ${ }^{1, *}$, Jan Versluis ${ }^{1}$, and Huib J. Bakker ${ }^{1}$ \\ ${ }^{1}$ Amolf, Ultrafast Spectroscopy, Science Park 104 Amsterdam, Netherlands \\ *cmoll@amolf.nl
}

\begin{abstract}
Spectroscopic studies of aqueous surfaces can provide a fundamental understanding of interfacial processes. These studies have largely focused on the $\mathrm{OH}$ stretch vibrations of water, which is unfortunate as the bending mode is an attractive feature to probe as it is relatively free of inter- and intramolecular couplings. However, the origin of the response of the bending mode is highly debated and has been assigned to either surface-specific or to bulk effects. Here, we study the bending mode of pure water and charged aqueous surfaces using heterodyne-detected vibrational sum frequency generation spectroscopy (HD-VSFG) to quantify the two effects. Our results show a low - $\left(1626 \mathrm{~cm}^{-1}\right)$ and a high - $\left(1656 \mathrm{~cm}^{-1}\right)$ frequency component which can be unambiguously assigned to an interfacial dipole and a bulk quadrupolar response, respectively. We thus demonstrate that probing the bending mode provides structural and quantitative information on both the surface and the bulk.
\end{abstract}

\section{Introduction}

The structure and dynamics of aqueous interfaces is of high relevance for many different scientific fields. ${ }^{1-9}$ Many important chemical and biological processes take place at aqueous interfaces, like for instance molecular recognition at bio-membranes, protein folding, and energy conversion and storage. ${ }^{10-15}$ The structural properties of the neat water/air interface ${ }^{16,17}$ and charged aqueous interfaces have been extensively studied with vibrational sum-frequency generation spectroscopy (VSFG) in the frequency region of the water $\mathrm{OH}$ stretch vibrations. ${ }^{11,18-20}$ The frequency region of the water bending vibrations has been much less investigated, even though this region can provide unique information on the hydrogen-bond structure, because, in contrast to the stretching mode, the water bending mode is hardly influenced by intermolecular coupling due to its small transition dipole moment. ${ }^{21-23}$ Furthermore, there is only one water bending mode per water molecule, thus strongly decreasing the effects of intramolecular coupling on the spectrum. ${ }^{21}$ The first measurements of the water bending mode of the neat water/air interface were performed with conventional VSFG, for which the response is proportional to the modulo squared of the second-order susceptibility $\left(\left|\chi^{(2)}\right|^{2}\right)$, by Vinaykin and Benderskii ${ }^{22}$. These authors reported the observation of an inhomogeneously broadened line shape centered at $1650 \mathrm{~cm}^{-1}$. They assigned the low- and high-frequency wing of this band to weakly hydrogen bonded water molecules sticking out of the surface, and to fully hydrogen-bonded interfacial water molecules, respectively. Furthermore, they found evidence for the presence of a large orientational inhomogeneity by measuring the $\left|\chi^{(2)}\right|^{2}$ spectrum with different polarization combinations (SSP and PPP) ${ }^{22}$. These findings have been confirmed by recent computational studies by Nagata et al. ${ }^{23}$, and $\mathrm{Ni}$ and Skinner $^{21}$, that showed that the different hydrogen-bonded water species correlate with different net orientations of the bending mode transition dipole moment.

Kundu et al. reported the first heterodyne detected VSFG (HD-VSFG) measurements of the neat water/air interface in the bending mode region. ${ }^{24}$ In this technique the real and imaginary part of the second-order susceptibility $\chi^{(2)}$ are determined. The measured $\operatorname{Im}\left[\chi^{(2)}\right]$ showed a single positive peak centered at $1650 \mathrm{~cm}^{-1}$. In the same work it was also reported that charging the interface negative with iodide ions leads to an enhancement of this signal. This observation strongly contrasts with observations in the $\mathrm{OH}$ stretching mode region, for which a change of the sign of the band is observed when the surface gets negatively charged. ${ }^{18,20}$ The accompanying calculations indicated that the observed $\operatorname{Im}\left[\chi^{(2)}\right]$ signal would originate mainly from a quadrupole response from the bulk. ${ }^{24}$ In the most recent intensity VSFG measurements of Benderskii ${ }^{15}$ it was found that the interfacial water molecules orient in different directions depending on the charge of the surface. This dependence of the orientation of the interfacial water molecules on the charge of the surface, suggests that the observed signal primarily results from a surface dipolar response. This explanation is consistent with the $\left|\chi^{(2)}\right|$ measurements of the group of Bonn and Nagata. ${ }^{25}$ The results of the $\left|\chi^{(2)}\right|^{2}$ measurements of the groups of Benderskii and Bonn and Nagata thus strongly disagree with the interpretation of the $\operatorname{Im}\left[\chi^{(2)}\right]$ measurements by Kundu et al. ${ }^{24}$, who reported that the signal in the bending mode region 
would mainly originate from a bulk quadrupolar response. Here we perform HD-VSFG measurements to investigate the sum-frequency response of aqueous systems in the frequency region of the bending mode. By measuring both the phase of the sum-frequency light and the effect of surface charge on the signal, we observe and distinguish both a dipolar contribution of the surface and a quadrupolar contribution of the bulk to the signal. We thus arrive at a consistent picture of the response of the water bending mode for different aqueous systems, and we can clear up the existing inconsistencies between earlier reports. $15,21,22,24,25$

\section{Results}

In Figure 1 we present $\left|\chi^{(2)}\right|^{2}$ (intensity VSFG) spectra of the neat water surface water/air (dark blue), the water/DTAB/air (darkcyan) and the water/SDS/air interface (magenta) in the frequency region of the water bend vibration. For pure water we

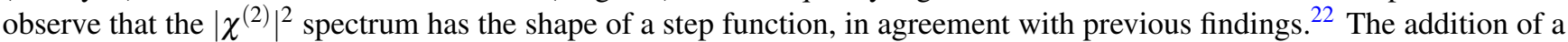
charged surfactant leads to strong changes of the response. Adding the positively charged DTAB leads to an enhancement and a redshift of the response, adding the negatively charged SDS leads to a redshift of the maximum of the SFG intensity.

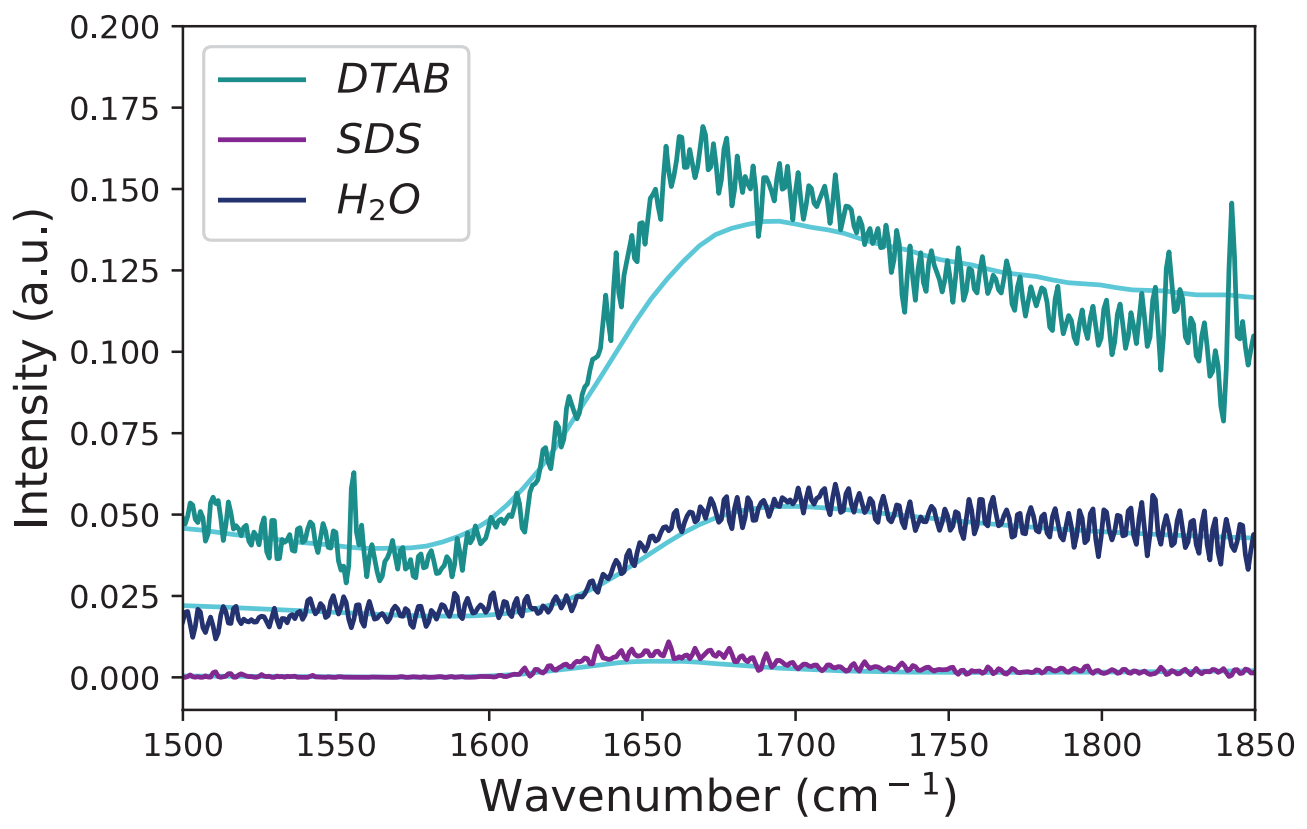

Figure 1. $\left|\chi^{(2)}\right|^{2}$ spectra of the neat water surface water/air (dark blue), the water/DTAB/air (darkcyan) and the water/SDS/air interface (magenta) in the frequency region of the water bend vibration. The concentration of the SDS and DTAB solution is 10 $\mathrm{mM}$ and the measurements are performed in SSP polarization configuration (S-SFG, S-VIS, P-IR). The solid curves are the result of model calculations, as described in the text. Note that the spectra are not offset with respect to each other, the signal observed for the DTAB solution is much stronger than that of pure water and the SDS solution. 
In Figure 2a the experimental $\operatorname{Im}\left[\chi^{(2)}\right]$ spectra of neat water (dark blue) in the frequency region of the water bend vibration is presented. We observe a symmetric positive peak centered at $1656 \mathrm{~cm}^{-1}$ that we assign to the water bending mode. The observed spectrum agrees with previous HD-VSFG measurements of the water bending mode of Kundu et al. ${ }^{24}$. We find that this $\operatorname{Im}\left[\chi^{(2)}\right]$ response can be fitted well with a single lorentzian curve (cyan). In Figure $2 \mathrm{~b}$ we show a comparison of the calculated $\operatorname{Im}\left[\chi^{(2)}\right]$ spectrum of neat water over a broad frequency region (cyan) combined with the experimental data (dark blue) in the frequency region of the water bending mode. In addition to the water bending mode, the model includes the resonances of the $\mathrm{OH}$ stretch vibrations of hydrogen-bonded and non-hydrogen-bonded water molecules (red dotted line, $\chi_{\sigma, O H}$ ). The $\mathrm{OH}$ stretch band of the hydrogen-bonded water molecules is modeled with two lorentzian curves with maxima at $3250 \mathrm{~cm}^{-1}$ and $3450 \mathrm{~cm}^{-1}$, and the response of the non-hydrogen-bonded water molecules is modeled with a single lorentzian centered at $3700 \mathrm{~cm}^{-1}$. In addition to the resonances in the stretching mode region, we included a frequency-independent non-resonant background $\chi_{N R}$ (orange dotted). The comparison of the model and the experimental data shows that the resonances of the $\mathrm{OH}$ stretch band have a negligible influence on the observed $\operatorname{Im}\left[\chi^{(2)}\right]$ in the bending mode region. However, the $\operatorname{Re}\left[\chi^{(2)}\right]$ in the bending mode region does show significant contributions from both the different $\mathrm{OH}$ stretch vibrations $\chi_{\sigma, O H}$ and the non-resonant background $\chi_{N R}$, as illustrated in Figure $2 \mathrm{c}, \mathrm{d}$. The low-frequency wing of the real response of the stretching band (red dotted) adds a negative value to the real part of the response of the bending mode $\chi_{\delta}$ (green dotted). In Figure $2 \mathrm{~d}$ we show the $\operatorname{Re}\left[\chi^{(2)}\right]$ over a broad frequency range. This figure clearly shows that the $\chi_{\sigma}$ contribution also induces a slight tilt of the total $\operatorname{Re}\left[\chi^{(2)}\right]$ in the frequency region of the bending mode. From the analysis of Figure 2 it is clear that the observed step in the intensity SFG spectrum of pure water of Figure 1 can be well explained from the interference with the stretch vibrations. The $\operatorname{Re}\left[\chi_{\sigma}^{(2)}\right]$ of the stretch vibrations compensates the positive $\operatorname{Re}\left[\chi_{\delta}^{(2)}\right]$ of the bending vibration at frequencies $<1650 \mathrm{~cm}^{-1}$, and
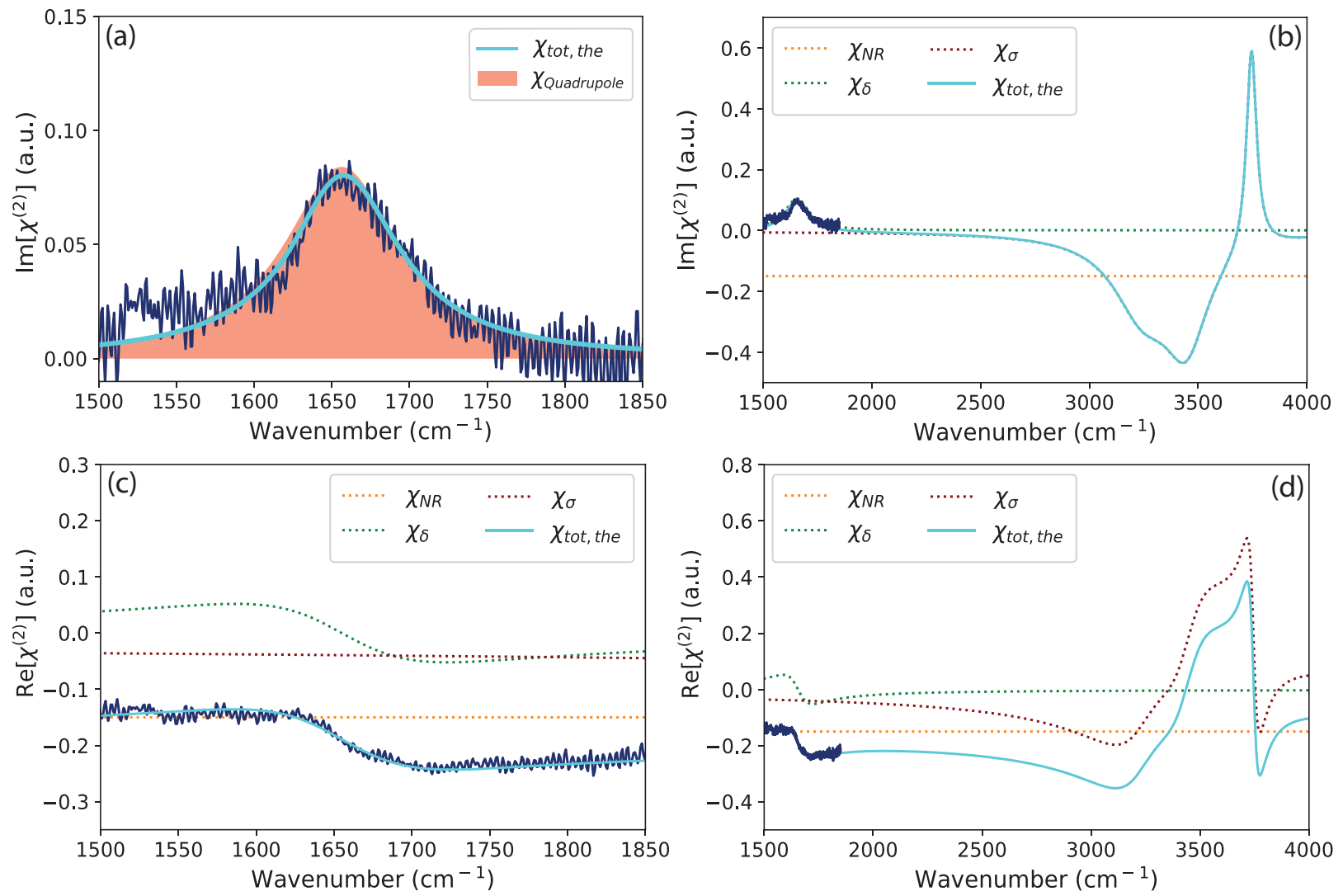

Figure 2. (a) $\operatorname{Im}\left[\chi^{(2)}\right]$ spectrum of neat water (dark blue) in the frequency region of the bending mode. The solid line represents the result of a fit, explained in the text. (b)-(d) modeled $\operatorname{Im}\left[\chi^{(2)}\right]$ and $\operatorname{Re}\left[\chi^{(2)}\right]$ spectra of neat water $\left(\chi_{\text {tot,the }}\right.$, cyan) compared to the experimental data (dark blue). The figures also show the decomposition of the total response $\chi_{\text {tot the }}$ in different contributions: the non-resonant background $\chi_{N R}$ (dotted orange), the bending mode response $\chi_{\delta}$ (dotted green), and the response of the $\mathrm{OH}$ stretch vibrations $\chi_{\sigma}$ (dotted red). 
enhances the negative $\operatorname{Re}\left[\chi_{\delta}^{(2)}\right]$ of the bending vibration at frequencies $>1650 \mathrm{~cm}^{-1}$. As a result, the total intensity $\left(\left|\chi^{(2)}\right|^{2}\right)$ shows a step-like shape.

In Figure 3, we present experimental $\operatorname{Im}\left[\chi^{(2)}\right]$ spectra of the water/DTAB/air (dark-cyan) and the water/SDS/air interface (magenta) in the frequency region of the water bend vibration. The $\operatorname{Im}\left[\chi^{(2)}\right]$ spectrum of the water/DTAB/air interface shows a positive band centered at $1640 \mathrm{~cm}^{-1}$. This band is stronger, broader and somewhat redshifted in comparison to the $\operatorname{Im}\left[\chi^{(2)}\right]$ spectrum of water/air. The $\operatorname{Im}\left[\chi^{(2)}\right]$ spectrum of the water/SDS/air interface shows a clear negative feature at lower frequencies. A this point it should be noted that we can exclude phase distortions in the HD-VSFG spectra, as the electric double layer (EDL) has a thickness $<10 \mathrm{~nm}$ for ionic strengths $>1 \mathrm{mM}^{26-28}$ The spectra observed for water/DTAB/air and water/SDS/air can be well explained from the presence of an additional low-frequency band in the $\operatorname{Im}\left[\chi^{(2)}\right]$ spectra that is negligibly present for neat water/air, and that has a different sign for the water/DTAB/air and the water/SDS/air interface. We thus find that the $\operatorname{Im}\left[\chi^{(2)}\right]$ response of neutral and surface-charged water can be well explained with two bands of which one changes sign depending on the sign of the surface charge. To check that the lower-frequency band truly depends on the sign of the surface charge, we also performed measurements with a different negatively charged surfactant sodium monododecyl phasphate (SMP) (see supporting figure 2 in the Supporting Information). These measurements also show the presence of a negative band centered at 1626 $\mathrm{cm}^{-1}$, thus confirming that this band results from the bending mode of water and the presence of negative charge at the surface. To quantify the different contributions to the $\operatorname{Im}\left[\chi^{(2)}\right]$ spectra we decompose the spectra observed for water/DTAB/air and water/SDS/air into lorentzian-shaped bands centered at $1656 \mathrm{~cm}^{-1}$ (blue) and $1626 \mathrm{~cm}^{-1}$ (red). The band at $1656 \mathrm{~cm}^{-1}$ does not depend on the charge of the surface and is assigned to the quadrupolar response of the bulk. The sign and amplitude of the $1626 \mathrm{~cm}^{-1}$ band are determined by the charge at the surface and can be assigned therefore to a dipolar surface contribution. The exact frequencies of the dipolar and quadrupolar contributions are extracted from the fitting procedure. For neat water we find that the experimental data can be well described with a single lorentzian line that only represents the quadrupolar contribution of the bulk (Figure 2). In our fitting procedure for the water/DTAB/air and water/SDS/air (10 mM) interfaces presented in Figs. $3 \mathrm{a}, \mathrm{b}$ we use the same quadrupolar contribution. In the Supplementary Information we show the measured $\operatorname{Re}\left[\chi^{(2)}\right]$ responses of water/DTAB/air and water/SDS/air, and the decomposition of the $\operatorname{Im}\left[\chi^{(2)}\right]$ and $\operatorname{Re}\left[\chi^{(2)}\right]$ spectra (Supporting Information Figures S3 (DTAB) and S4 (SDS)), as was done for neat water/air, which was shown in Figure 2. The thus obtained $\operatorname{Re}\left[\chi^{(2)}\right]$ and $\operatorname{Im}\left[\chi^{(2)}\right]$ responses account very well for the observed effects in the $\left|\chi^{(2)}\right|^{2}$ spectra of the three studied systems shown in Figure 1. The solid lines in Figure 1 are the result of the complete analysis of the $\chi^{(2)}$, as is shown in Figure 2, and Figures S3 and S4 of the SI, and include the interference effect of the bending mode with the stretch vibrations and the nonresonant background, and the quadrupolar bulk and dipolar interface contributions to the bending mode response. We find that for water/DTAB/air and water/SDS/air the $\operatorname{Re}\left[\chi^{(2)}\right]$ response is again strongly affected by the interference with the response of the $\mathrm{OH}$ stretch vibrations, as was the case for neat water.

For the positively charged DTAB/air surface the interfacial water molecules are oriented with their hydrogen atoms towards the bulk. For the stretch vibration this orientation leads to a negative contribution to $\operatorname{Im}\left[\chi^{(2)}\right]$, because for the stretch vibration
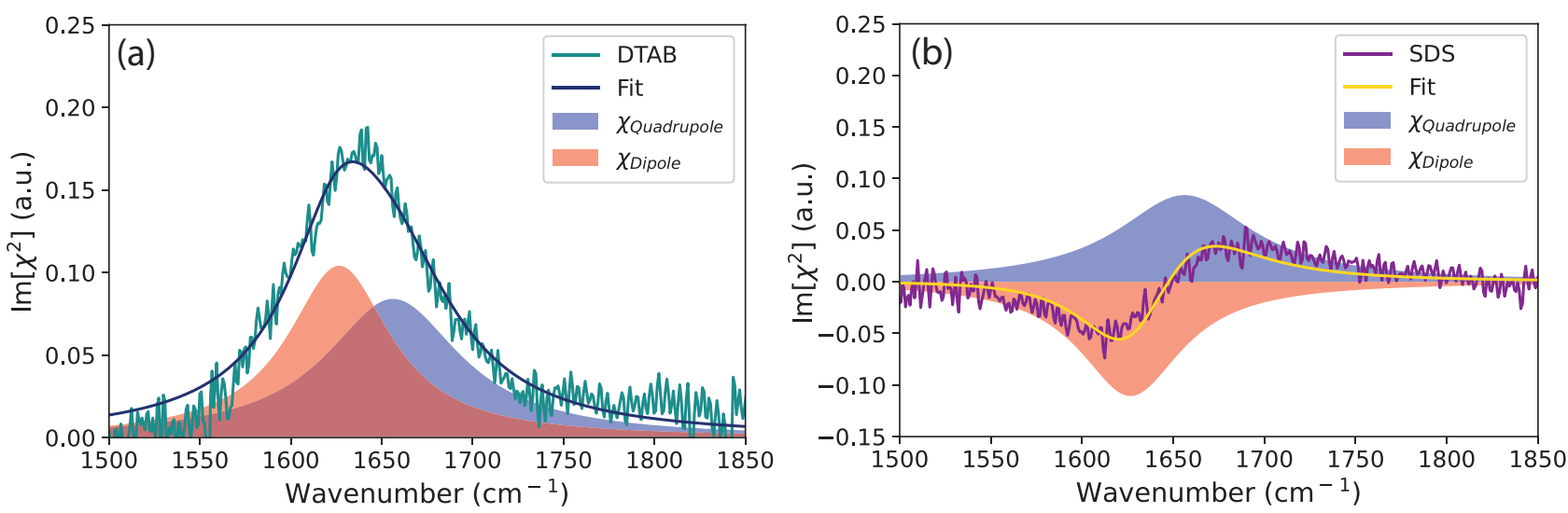

Figure 3. Spectral decomposition of the $\operatorname{Im}\left[\chi^{(2)}\right]$ spectra of the water/DTAB/air (dark cyan) and the water/SDS/air interface (magenta), each modeled with two lorentzian-shaped spectral components. The two lorentzian-shaped components are assigned to the quadrupolar contribution of the bulk (blue, centered at $1656 \mathrm{~cm}^{-1}$ ), and to the surface dipolar contribution (red, centered at $1626 \mathrm{~cm}^{-1}$ ), of the water bending mode. The DTAB and SDS solution have a concentration of $10 \mathrm{mM}$. 

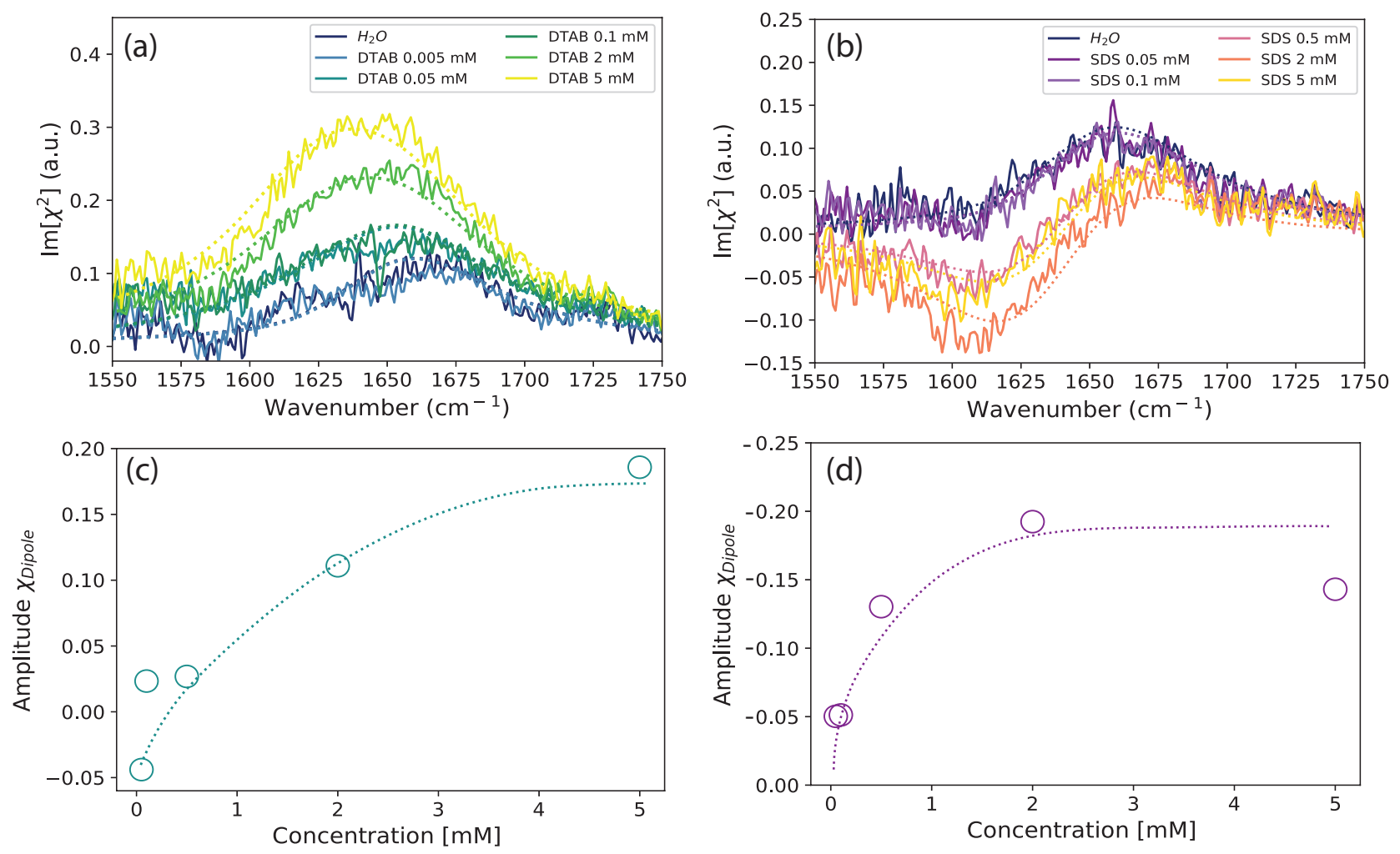

Figure 4. The $\operatorname{Im}\left[\chi^{(2)}\right]$ spectra of the water/DTAB/air (4(a)) and the water/SDS/air (4(b)) interface at different concentrations in the range of 0.05 to $5 \mathrm{mM}$ including fits presented with dashed lines. $4((\mathrm{c})-(\mathrm{d}))$ show the absolute amplitude of the dipole contribution extracted from the fitting procedure in respect to the concentration of DTAB 4(c) and SDS 4(d).

the dipole and the polarizability have the same dependence on the phase of the stretch vibration (when the hydrogen atoms move away from the oxygen atom, the dipole moment increases and the size and polarizability of the water molecules also increase). For the bending vibration the dipole moment and polarizability have an opposite dependence on the phase of the bending vibration (when the atoms move together, the dipole moment increases while the size and polarizability of the water molecule decreases). As a result, the surface dipolar contribution to the $\operatorname{Im}\left[\chi^{(2)}\right]$ of the bending mode is positive for water molecules with their hydrogen atoms oriented towards the bulk. For the negatively charged water/SDS/air interface, the water molecules have their hydrogen atoms oriented towards the surface and the surface dipolar contribution to the $\operatorname{Im}\left[\chi^{(2)}\right]$ of the bending mode is negative.

In Figure 4 we present the $\operatorname{Im}\left[\chi^{(2)}\right]$ spectra of water/DTAB/air (4a) and water/SDS/air (4b) at different concentrations in the range of $0 \mathrm{mM}$ to $5 \mathrm{mM}$. For water/DTAB/air we observe that the positive peak centered at $1656 \mathrm{~cm}^{-1}$ assigned to the water bending mode is increasing with increasing concentration of DTAB. Furthermore, we observe a broadening and a slight red shift of the $\operatorname{Im}\left[\chi^{(2)}\right]$ spectra. Increasing the SDS concentration leads to an ingrowth of a negative peak at lower frequencies. These observations confirm the assignment of the band at $1626 \mathrm{~cm}^{-1}$ to a surface dipolar contribution. In the case of DTAB, the positive surface charge is increasing with increasing concentration, thus causing an enhanced ordering of the interfacial water molecules with their hydrogen atoms towards the bulk, which leads to an increase of a positive dipolar band at $1626 \mathrm{~cm}^{-1}$ to $\operatorname{Im}\left[\chi^{(2)}\right]$. The increase of the concentration of SDS increases the negative charge density at the surface, thereby inducing an enhanced orientation of the water molecules with their hydrogen atoms towards the surface, and thus to an increase of a negative dipolar contribution at $1626 \mathrm{~cm}^{-1}$ to the $\operatorname{Im}\left[\chi^{(2)}\right]$ response.

To quantify the results, we decompose the responses measured at different concentrations of DTAB and SDS in two lorentzian bands centered at $1626 \mathrm{~cm}^{-1}$ and $1656 \mathrm{~cm}^{-1}$, for the dipole and quadrupol contribution, respectively. While keeping the quadrupolar contribution and the width of the dipolar contribution constant, we allow the amplitude of the dipole contribution to change with varying concentration. The fits are shown in Figure 4 (a-b) with dashed lines. The decompositions for the different concentrations are presented in the Supporting Information (Supporting Figures 5 (DTAB) and 6 (SDS)). In 
Figure 4c,d we present the amplitude of the dipolar band extracted from the fitting procedure as a function of the surfactant concentration. For both DTAB and SDS, the amplitude of the dipolar band increases nonlinearly with concentration, showing saturation behavior at a bulk concentration of a few $\mathrm{mM}$. From the fitting procedure we find that a a surfactant concentration of $10 \mathrm{mM}$ the absolute ratio of the amplitudes of the dipolar interfacial and the quadrupolar bulk contribution is 1:0.7, both for the water/DTAB/air interface and the water/SDS/air interface. It is also seen that at zero surfactant concentration the dipolar contribution is negligible.

\section{Discussion}

Recently Ahmed et al. ${ }^{29}$ reported HD-VSFG measurements of water with charged surfactants and lipid monolayer/water interfaces in which they did not find evidence for an interfacial dipole contribution of the water bending mode. $\operatorname{Their} \operatorname{Im}\left[\chi^{(2)}\right]$ spectra show a positive band, independent on the sign of the charge of the headgroup. Therefore, Ahmed et al. argued that the $\operatorname{Im}\left[\chi^{(2)}\right]$ response of the water bending mode arises only from a quadrupolar response, even for charged surfaces. ${ }^{29}$ At first sight this finding appears to disagree with the results of Figure 3. However, the measurements of Ahmed et al. were performed with a surfactant concentration of only $0.1 \mathrm{mM}$, while the measurements shown in Figure 3 are performed with a surfactant concentration of $10 \mathrm{mM}$. As is clearly illustrated in Figure 4, for a surfactant concentration of $0.1 \mathrm{mM}$, the dipolar contribution is negligibly small. At these low concentrations the contribution of the dipolar surface concentration merely induces a small change in amplitude and shift in frequency of the overall measured $\operatorname{Im}\left[\chi^{(2)}\right]$ response. An interesting observation is that the dipolar contribution has a significantly lower frequency than the quadrupolar contribution. For the bending mode, the vibrational frequency increases with increasing strength of the hydrogen-bond interaction. ${ }^{22}$ Hence, the lower frequency of the dipolar contribution indicates that the hydrogen bonds of the contributing water molecules are weaker than those of the water molecules giving rise to the quadrupolar contribution. This finding agrees with the fact that the molecules giving rise to the quadrupolar contribution are predominantly located in the bulk of the liquid, whereas the molecules giving rise to the dipolar contribution will be located at the surface. In summary, we performed heterodyne detected vibrational surface sum-frequency generation (HD-VSFG) measurements of pure water, and of aqueous solutions of ionic surfactants, i.e. of a dodecyltrimethylammonium bromide solution (DTAB), and of a sodium dodecyl sulfate solution (SDS) solution. We find that the sum-frequency generation response in the frequency region of the bending mode of water is strongly affected by the interference with the low-frequency wing of the $\mathrm{OH}$ stretch vibrations of water. The different contributions of the bending mode and the stretching modes can be distinguished and quantified.

By comparing the measured $\operatorname{Im}\left[\chi^{(2)}\right]$ spectra of differently charged water surfaces, we demonstrate the presence of a surface-specific dipolar response of the water bending mode centered at $1626 \mathrm{~cm}^{-1}$. This response changes sign when the sign of the surface charge changes, and increases with increasing surface charge, i.e. increasing concentration of ionic surfactant. In addition to this dipolar surface contribution, the bending mode of water shows a quadrupolar bulk contribution centered at 1656 $\mathrm{cm}^{-1}$. This contribution is not surface-charge dependent and dominates the $\operatorname{Im}\left[\chi^{(2)}\right]$ response of neat water in the frequency region of the bending mode. We thus demonstrate that HD-VSFG involving the bending mode of the water molecules can provide important information on the hydrogen-bond structure of both the surface and the bulk of aqueous solutions.

\section{Methods}

The technique of HD-VSFG has been developed and successfully used by several groups..$^{20,30,31}$ Here we give a brief description of our implementation of HD-VSFG. A more detailed description of the setup can be found in previous work. ${ }^{32}$ We use an amplified Ti:Sapphire laser system $(1 \mathrm{kHz}, 35 \mathrm{fs}, 6.5 \mathrm{~mJ} / \mathrm{pulse})$ to generate a narrow $800 \mathrm{~nm}$ beam and a tunable, broadband mid-infrared beam. The two beams are spatially and temporally overlapped at the surface of a gold mirror to generate light at the sum-frequency that serves as a local oscillator (LO-SFG). The $800 \mathrm{~nm}$ beam (s-polarized), the IR beam (p-polarized) and the LO-SFG signal (s-polarized) are all sent onto a concave mirror. Before the beams are focused on the sample surface, the LO-SFG signal is passing through a silica plate to delay it in time ( $\sim 1.6 \mathrm{ps})$. The $800 \mathrm{~nm}$ and IR beam are generating a second SFG signal at the sample surface. Both the SFG signal of the sample and the LO-SFG signal are sent into a spectrometer and detected with a thermoelectrically cooled charged-coupled device (CCD, Princeton Instruments). From the interference spectrum of the two SFG signals the real (Re) and the imaginary $(\operatorname{Im}) \chi^{(2)}$ spectra can be extracted, providing direct information on the orientation of the vibrational transition dipole moments, and thus on the absolute orientation of the molecules at the surface. ${ }^{33}$ We also perform a reference experiment in which the sample is replaced by a z-cut quartz crystal. The typical acquisition time of a single HD-VSFG spectrum is 600 seconds. The water bending mode has a small transition dipole moment and therefore yields a $\sim 5$ times smaller signal than the $\mathrm{OH}$ stretching modes. Therefore, the measurement of a clean signal confronts us with several technical challenges. In particular, in the frequency region of the water bending mode an etaloning effect occurs on the CCD camera, which introduces a systematic modulation on the signal. A detailed explanation of how we were able to remove this systematic modulation can be found in the Supporting Information (Noise reduction and 
measurement procedure and Supporting figure 1). For all measurements we used water from a Millipore Nanopure system $(18,2 \mathrm{Mcm})$. Both DTAB and SDS are purchased from Sigma Aldrich and used without further purification. In all experiments we observe a small frequency-independent positive offset that we assign to background noise of which the amplitude varies between different experiments. We extract the amplitude of this background noise from our fitting procedure. In all presented data this background noise has been subtracted.

\section{References}

1. Nihonyanagi, S., Yamaguchi, S. \& Tahara, T. Ultrafast Dynamics at Water Interfaces Studied by Vibrational Sum Frequency Generation Spectroscopy. Chem. Rev. 117, 10665-10693, DOI: 10.1021/acs.chemrev.6b00728 (2017).

2. Koinuma, H. et al. Ultrafast Dynamics at Water Interfaces. Science 313, 1945-1948 (2006).

3. Cyran, J. D., H. G. Backus, E., Nagata, Y. \& Bonn, M. Structure from Dynamics: Vibrational Dynamics of Interfacial Water as a Probe of Aqueous Heterogeneity. The J. Phys. Chem. B 122, 3667-3679, DOI: 10.1021/acs.jpcb.7b10574 (2018).

4. Strazdaite, S., Versluis, J., Backus, E. H. G. \& Bakker, H. J. Enhanced ordering of water at hydrophobic surfaces. The J. Chem. Phys. 140, 54711, DOI: 10.1063/1.4863558 (2014).

5. Chen, X., Yang, T., Kataoka, S. \& S. Cremer, P. Specific Ion Effects on Interfacial Water Structure near Macromolecules. J. Am. Chem. Soc. 129, 12272-12279, DOI: 10.1021/ja073869r (2007).

6. Xiong, W., Laaser, J. E., Mehlenbacher, R. D. \& Zanni, M. T. Adding a dimension to the infrared spectra of interfaces using heterodyne detected 2D sum-frequency generation (HD 2D SFG) spectroscopy. Proc. Natl. Acad. Sci. 108, 20902-20907, DOI: 10.1073/PNAS.1115055108 (2011).

7. Hsieh, C.-S. et al. Aqueous Heterogeneity at the Air/Water Interface Revealed by 2D-HD-SFG Spectroscopy. Angewandte Chemie Int. Ed. 53, 8146-8149, DOI: https://doi.org/10.1002/anie.201402566 (2014).

8. Tyrode, E., Sengupta, S. \& Sthoer, A. Identifying Eigen-like hydrated protons at negatively charged interfaces. Nat. Commun. 11, 1-7, DOI: 10.1038/s41467-020-14370-5 (2020).

9. Singh, P. C., Nihonyanagi, S., Yamaguchi, S. \& Tahara, T. Interfacial water in the vicinity of a positively charged interface studied by steady-state and time-resolved heterodyne-detected vibrational sum frequency generation. The J. Chem. Phys. 141, 18C527, DOI: 10.1063/1.4897265 (2014).

10. Strazdaite, S., Meister, K. \& Bakker, H. J. Orientation of polar molecules near charged protein interfaces. Phys. Chem. Chem. Phys. 18, 7414-7418, DOI: 10.1039/c5cp06372h (2016).

11. Wen, Y. C. et al. Unveiling Microscopic Structures of Charged Water Interfaces by Surface-Specific Vibrational Spectroscopy. Phys. Rev. Lett. 116, 1-5, DOI: 10.1103/PhysRevLett.116.016101 (2016).

12. Lo Nostro, P. \& Ninham, B. W. Hofmeister phenomena: An update on ion specificity in biology. Chem. Rev. 112, 2286-2322, DOI: $10.1021 / \mathrm{cr} 200271 \mathrm{j}$ (2012).

13. Zhang, L., Yang, Y., Kao, Y. T., Wang, L. \& Zhong, D. Protein hydration dynamics and molecular mechanism of coupled water-protein fluctuations. J. Am. Chem. Soc. 131, 10677-10691, DOI: 10.1021/ja902918p (2009).

14. Michael F. Toney, Jason N. Howard, Jocelyn Richer, Gary L. Borges, Joseph G. Gordon, Owen R. Melroy, David G. Wiesler, D. Y. \&. L. B. S. (C) 1991 Nature Publishing Group . Nature 368, 444-446 (1994).

15. Dutta, C., Mammetkuliyev, M. \& Benderskii, A. V. Re-orientation of water molecules in response to surface charge at surfactant interfaces. J. Chem. Phys. 151, DOI: 10.1063/1.5066597 (2019).

16. Du, Q., Superfine, R., Freysz, E. \& Shen, Y. R. Vibrational spectroscopy of water at the vapor/water interface. Phys. Rev. Lett. 70, 2313-2316, DOI: 10.1103/PhysRevLett.70.2313 (1993). arXiv:1011.1669v3.

17. Tainter, C. J., Ni, Y., Shi, L. \& Skinner, J. L. Hydrogen bonding and oh-stretch spectroscopy in water: Hexamer (Cage), liquid surface, liquid, and ice. J. Phys. Chem. Lett. 4, 12-17, DOI: 10.1021/jz301780k (2013).

18. Tian, C., Byrnes, S. J., Han, H. L. \& Shen, Y. R. Surface propensities of atmospherically relevant ions in salt solutions revealed by phase-sensitive sum frequency vibrational spectroscopy. J. Phys. Chem. Lett. 2, 1946-1949, DOI: 10.1021/ jz200791c (2011).

19. Tian, C. S. \& Shen, Y. R. Structure and charging of hydrophobic material/water interfaces studied by phase-sensitive sum-frequency vibrational spectroscopy. Proc. Natl. Acad. Sci. United States Am. 106, 15148-15153, DOI: 10.1073/pnas. 0901480106 (2009). 
20. Nihonyanagi, S., Yamaguchi, S. \& Tahara, T. Direct evidence for orientational flip-flop of water molecules at charged interfaces: A heterodyne-detected vibrational sum frequency generation study. J. Chem. Phys. 130, DOI: 10.1063/1. 3135147 (2009).

21. Ni, Y. \& Skinner, J. L. IR and SFG vibrational spectroscopy of the water bend in the bulk liquid and at the liquid-vapor interface, respectively. J. Chem. Phys. 143, DOI: 10.1063/1.4923462 (2015).

22. Vinaykin, M. \& Benderskii, A. V. Vibrational sum-frequency spectrum of the water bend at the air/water interface. J. Phys. Chem. Lett. 3, 3348-3352, DOI: 10.1021/jz3014776 (2012).

23. Nagata, Y. et al. Water bending mode at the water-vapor interface probed by sum-frequency generation spectroscopy: A combined molecular dynamics simulation and experimental study. J. Phys. Chem. Lett. 4, 1872-1877, DOI: 10.1021/ jz400683v (2013).

24. Kundu, A. et al. Bend Vibration of Surface Water Investigated by Heterodyne-Detected Sum Frequency Generation and Theoretical Study: Dominant Role of Quadrupole. J. Phys. Chem. Lett. 7, 2597-2601, DOI: 10.1021/acs.jpclett.6b00657 (2016).

25. Seki, T. et al. Unveiling Heterogeneity of Interfacial Water through the Water Bending Mode. J. Phys. Chem. Lett. 10, 6936-6941, DOI: 10.1021/acs.jpclett.9b02748 (2019).

26. E. Gragson, D. \& L. Richmond, G. Investigations of the Structure and Hydrogen Bonding of Water Molecules at Liquid Surfaces by Vibrational Sum Frequency Spectroscopy. The J. Phys. Chem. B 102, 3847-3861, DOI: 10.1021/jp9806011 (1998).

27. Ohno, P. E., Wang, H. F., Paesani, F., Skinner, J. L. \& Geiger, F. M. Second-Order Vibrational Lineshapes from the Air/Water Interface. J. Phys. Chem. A 122, 4457-4464, DOI: 10.1021/acs.jpca.8b02802 (2018).

28. Ohno, P. E., Wang, H. F. \& Geiger, F. M. Second-order spectral lineshapes from charged interfaces. Nat. Commun. 8, 1-9, DOI: 10.1038/s41467-017-01088-0 (2017).

29. Ahmed, M., Nihonyanagi, S., Kundu, A., Yamaguchi, S. \& Tahara, T. Resolving the Controversy over Dipole versus Quadrupole Mechanism of Bend Vibration of Water in Vibrational Sum Frequency Generation Spectra. The J. Phys. Chem. Lett. 9123-9130, DOI: 10.1021/acs.jpclett.0c02644 (2020).

30. Ostroverkhov, V., Waychunas, G. A. \& Shen, Y. R. New information on water interfacial structure revealed by phasesensitive surface spectroscopy. Phys. Rev. Lett. 94, 2-5, DOI: 10.1103/PhysRevLett.94.046102 (2005).

31. Yamaguchi, S. \& Tahara, T. Heterodyne-detected electronic sum frequency generation: "Up" versus "down" alignment of interfacial molecules. J. Chem. Phys. 129, DOI: 10.1063/1.2981179 (2008).

32. Moll, C. J., Meister, K., Kirschner, J. \& Bakker, H. J. Surface Structure of Solutions of Poly(vinyl alcohol) in Water. J. Phys. Chem. B 122, 10722-10727, DOI: 10.1021/acs.jpcb.8b08374 (2018).

33. Shen, Y. R. Phase-Sensitive Sum-Frequency Spectroscopy. Annu. Rev. Phys. Chem. 64, 129, DOI: 10.1146/ annurev-physchem-040412-110110 (2013).

\section{Acknowledgements}

This work is part of the research program of the Netherlands Organization for Scientific Research (NWO) and was performed at the research institute AMOLF.

\section{Author contributions statement}

C.J. Moll, J. Versluis designed research idea; C.J. Moll performed the experiments; C.J. Moll, J. Versluis, H.J. Bakker analyzed and discussed the data; and C.J. Moll, J. Versluis, H.J. Bakker wrote the paper.

\section{Additional information}

The authors declare no competing interest. 
Figures

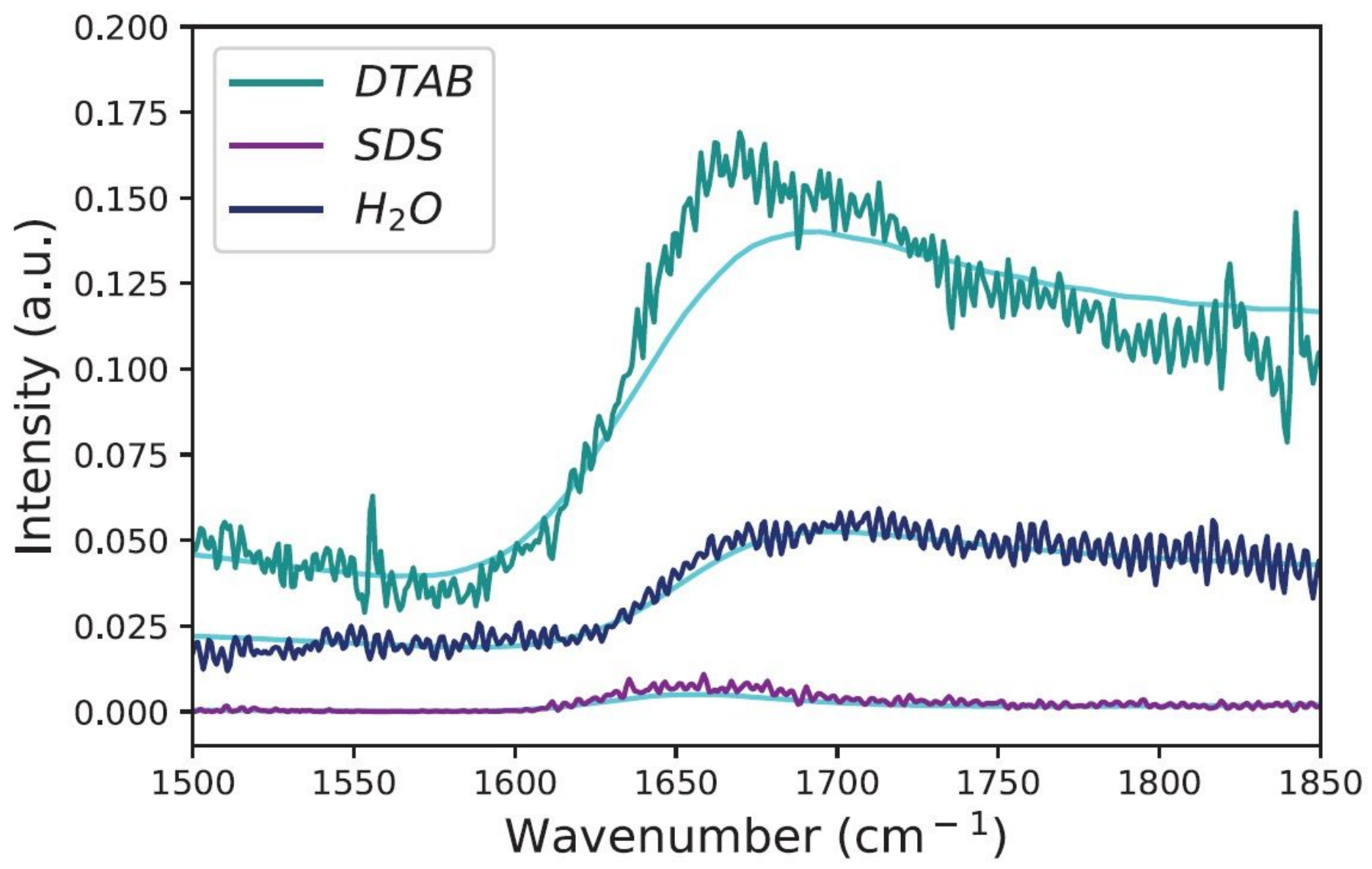

Figure 1

$|x(2)| 2$ spectra of the neat water surface water/air (dark blue), the water/DTAB/air (darkcyan) and the water/SDS/air interface (magenta) in the frequency region of the water bend vibration. The concentration of the SDS and DTAB solution is $10 \mathrm{mM}$ and the measurements are performed in SSP polarization configuration (S-SFG, S-VIS, P-IR). The solid curves are the result of model calculations, as described in the text. Note that the spectra are not offset with respect to each other, the signal observed for the DTAB solution is much stronger than that of pure water and the SDS solution. 

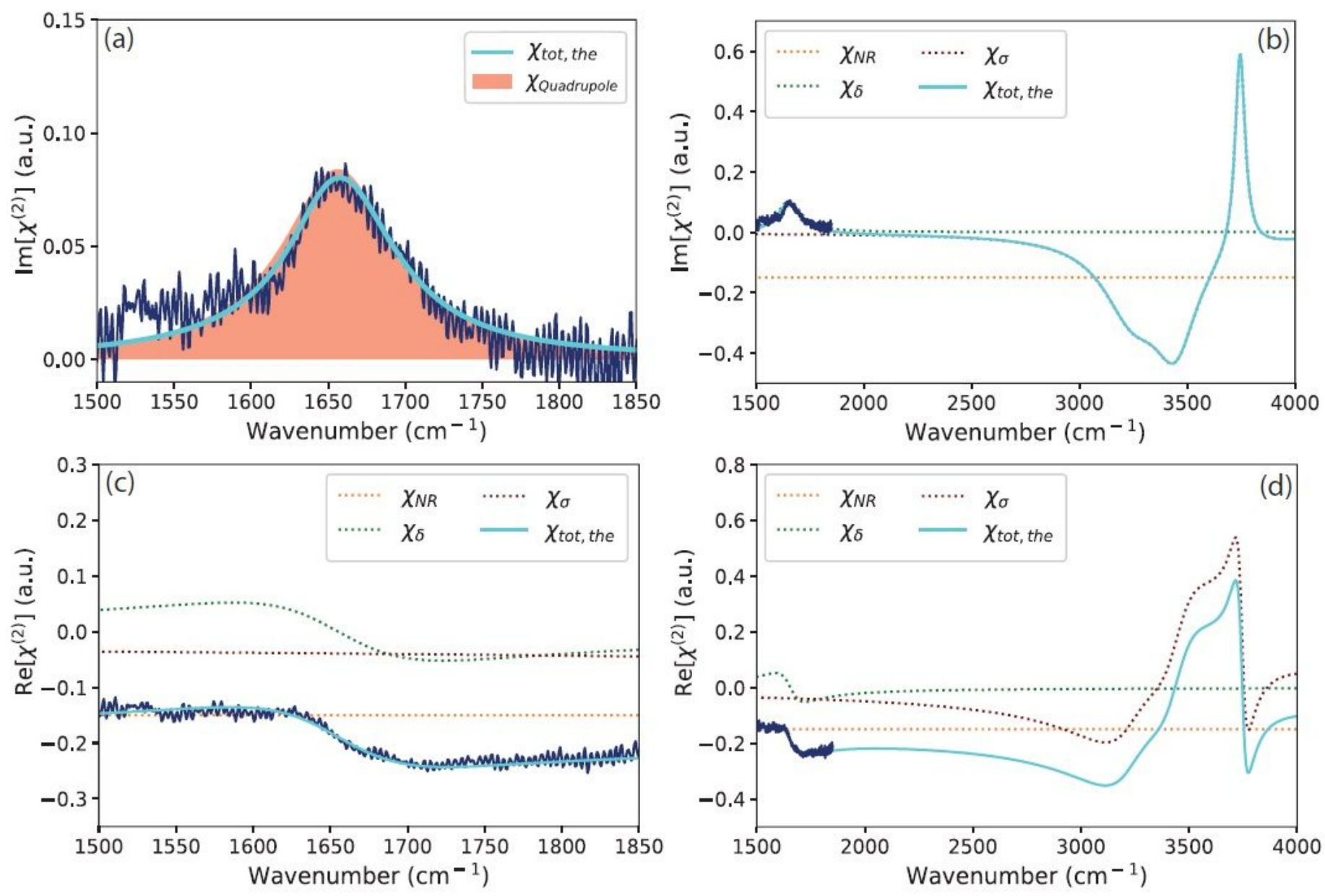

Figure 2

(a) $\operatorname{Im}[x(2)]$ spectrum of neat water (dark blue) in the frequency region of the bending mode. The solid line represents the result of a fit, explained in the text. (b)-(d) modeled $\operatorname{Im}[x(2)]$ and $\operatorname{Re}[x(2)]$ spectra of neat water (xtot;the, cyan) compared to the experimental data (dark blue). The figures also show the decomposition of the total response xtot,the in different contributions: the non-resonant background cNR (dotted orange), the bending mode response cd (dotted green), and the response of the $\mathrm{OH}$ stretch vibrations cs (dotted red). 

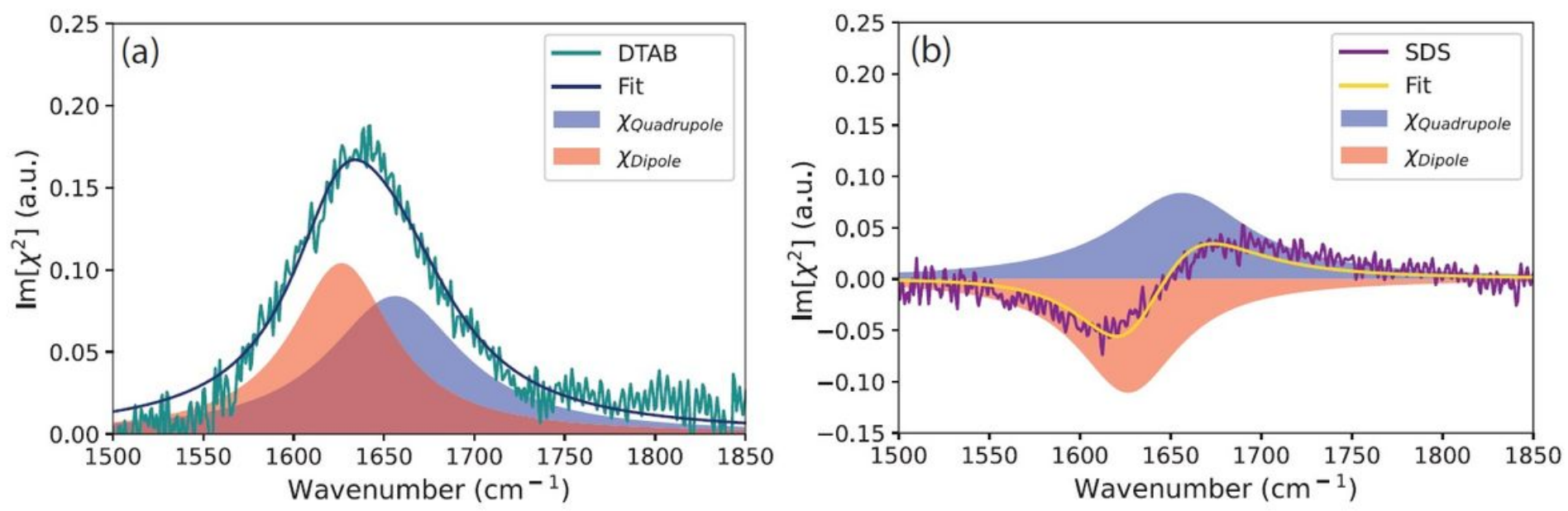

Figure 3

Spectral decomposition of the $\operatorname{Im}[x(2)]$ spectra of the water/DTAB/air (dark cyan) and the water/SDS/air interface (magenta), each modeled with two lorentzian-shaped spectral components. The two lorentzianshaped components are assigned to the quadrupolar contribution of the bulk (blue, centered at $1656 \mathrm{~cm}$ 1), and to the surface dipolar contribution (red, centered at $1626 \mathrm{~cm}-1$ ), of the water bending mode. The DTAB and SDS solution have a concentration of $10 \mathrm{mM}$.
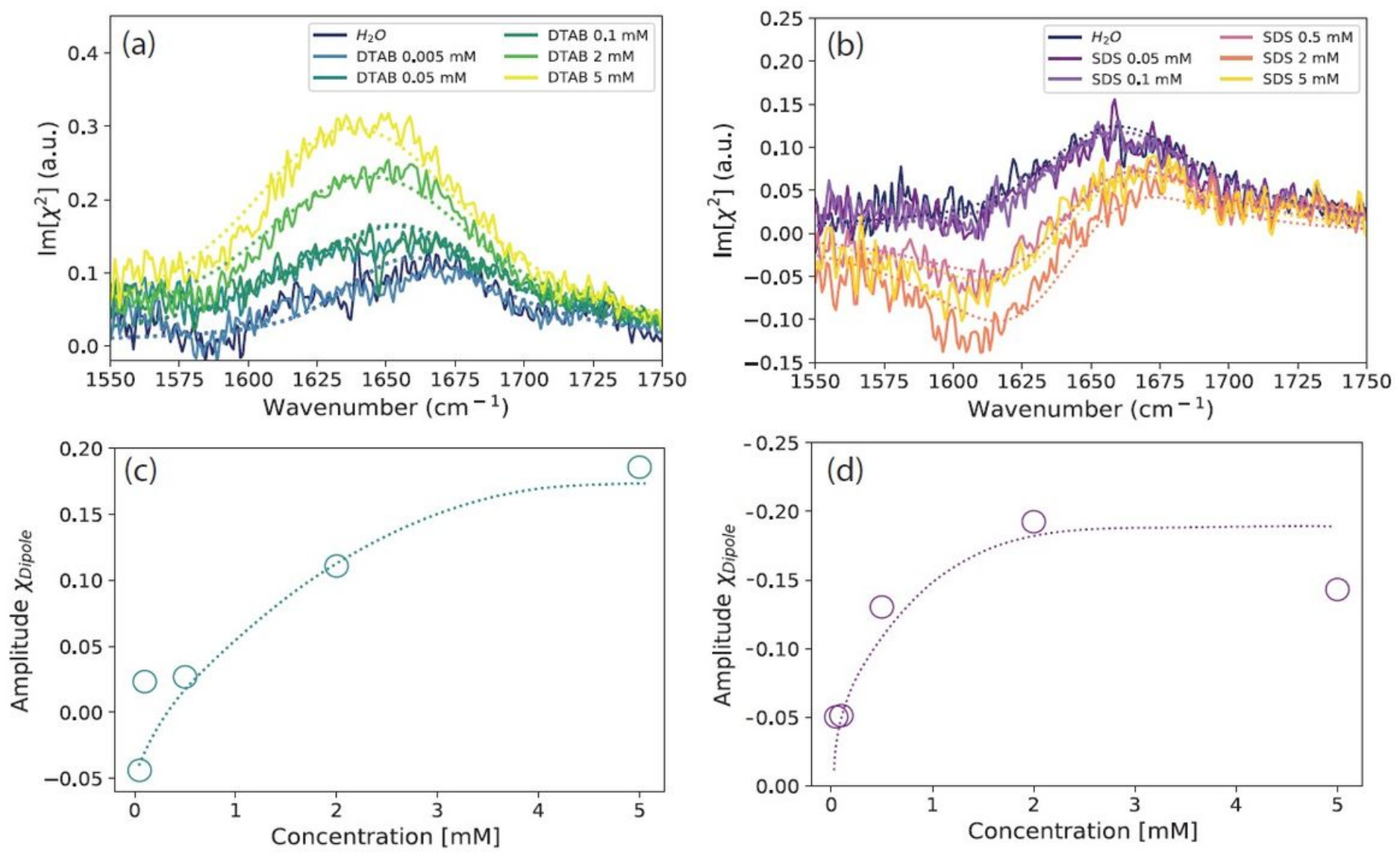

Figure 4 
The $\operatorname{Im}[x(2)]$ spectra of the water/DTAB/air (4(a)) and the water/SDS/air (4(b)) interface at different concentrations in the range of 0.05 to $5 \mathrm{mM}$ including fits presented with dashed lines. $4((\mathrm{c})$-(d)) show the absolute amplitude of the dipole contribution extracted from the fitting procedure in respect to the concentration of DTAB 4(c) and SDS 4(d).

\section{Supplementary Files}

This is a list of supplementary files associated with this preprint. Click to download.

- Supportinglnformation.pdf 